\title{
Observation of Dirac Holes and Electrons in a Topological Insulator
}

\author{
A. A. Taskin, Zhi Ren, Satoshi Sasaki, Kouji Segawa, and Yoichi Ando \\ Institute of Scientific and Industrial Research, Osaka University, Ibaraki, Osaka 567-0047, Japan
}

\begin{abstract}
We show that in the new topological-insulator compound $\mathrm{Bi}_{1.5} \mathrm{Sb}_{0.5} \mathrm{Te}_{1.7} \mathrm{Se}_{1.3}$ one can achieve a surfaced-dominated transport where the surface channel contributes up to $70 \%$ of the total conductance. Furthermore, it was found that in this material the transport properties sharply reflect the time dependence of the surface chemical potential, presenting a sign change in the Hall coefficient with time. We demonstrate that such an evolution makes us observe both Dirac holes and electrons on the surface, which allows us to reconstruct the surface band dispersion across the Dirac point.
\end{abstract}

PACS numbers: 73.25.+i, 71.18.+y, 73.20.At, 72.20.My

The three-dimensional (3D) topological insulator (TI) hosts a metallic surface state that emerges due to a nontrivial $\mathrm{Z}_{2}$ topology of the bulk valence band [1, 2]. This peculiar surface state offers a new playground for studying the physics of quasiparticles with unusual dispersions, such as Dirac or Majorana fermions [3, 4]. However, most of the known TI materials are poor insulators in their bulk, hindering transport studies of the expected novel surface properties [3, 4]. Last year, we discovered that the TI material $\mathrm{Bi}_{2} \mathrm{Te}_{2} \mathrm{Se}$ (BTS) presents a high resistivity exceeding $1 \Omega \mathrm{cm}$ [5], which made it possible to clarify both the surface and bulk transport channels in detail. Also, we found that in our BTS sample the surface channel accounts for $\sim 6 \%$ of the total conductance. For this BTS compounds, Xiong et al. recently reported a higher resistivity in the range of $5-6 \Omega \mathrm{cm}$, together with pronounced surface quantum oscillations which possibly signify fractional-filling states [ $[6]$. Since the source of the residual bulk carriers in BTS is the acceptor states [5], reducing the number of anti-site defects working as acceptors in this promising material is an important challenge for the advancement of the TI research.

In this work, we tried to optimize the composition of BTS by reducing the Te/Se ratio and introducing some $\mathrm{Sb}$ into Bi positions [7], while keeping the ordering of the chalcogen layers as in BTS (Fig. 1(a), [8]). The X-ray powder diffraction patterns shown in Fig. 1(b) demonstrate that the chalcogen ordering is still present in $\mathrm{Bi}_{1.5} \mathrm{Sb}_{0.5} \mathrm{Te}_{1.7} \mathrm{Se}_{1.3}$ (BSTS), and we focus on this compound in this Letter. We found that in BSTS one can achieve an even larger surface contribution (up to 70\%) than in BTS. We also found that the surface state of BSTS changes with time, and, intriguingly, we observed quantum oscillations from Dirac holes, the negative energy state of the Dirac fermions, as well as those from Dirac electrons in the same sample at different time points. We show that this time evolution can be used to reconstruct the surface band structure across the Dirac point, providing a unique way to determine the dispersion relation of the surface state.

Single crystals of BSTS were grown by melting high purity $(6 \mathrm{~N})$ elements of $\mathrm{Bi}, \mathrm{Sb}, \mathrm{Te}$, and Se with a mo-
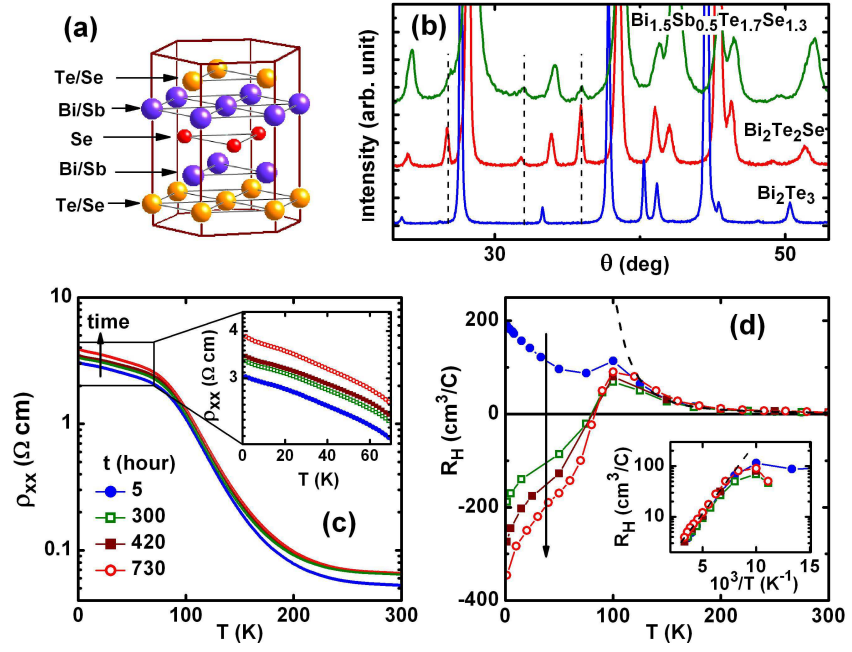

FIG. 1: (Color online) (a) Basic structure unit of $\mathrm{Bi}_{1.5} \mathrm{Sb}_{0.5} \mathrm{Te}_{1.7} \mathrm{Se}_{1.3}$ (BSTS). (b) Comparison of the X-ray powder diffraction patterns of BSTS, $\mathrm{Bi}_{2} \mathrm{Te}_{2} \mathrm{Se}$, and $\mathrm{Bi}_{2} \mathrm{Te}_{3}$. Dashed lines indicate the positions of the peaks characteristic of the ordering of Se and Te (Te/Se) layers. (c) Temperature dependence of $\rho_{x x}$ measured repeatedly in time in a cleaved $30-\mu \mathrm{m}$-thick BSTS sample. (d) Temperature dependence of the low-field $R_{H}$, presenting a sign change with time. Dashed line represents the Arrhenius-law fitting to the data above 150 $\mathrm{K}$, which is also shown in the inset.

lar ratio of 1.5:0.5:1.7:1.3 at $850^{\circ} \mathrm{C}$ for $48 \mathrm{~h}$ in evacuated quartz tubes, followed by cooling to room temperature over one week. For transport measurements, crystals were cut along the principal axes, and cleaved just before the measurements. Ohmic contacts were prepared by using room-temperature cured silver paste. The resistivity $\rho_{x x}$ and the Hall resistivity $\rho_{y x}$ were measured simultaneously by a standard six-probe method [9] by sweeping the magnetic field $B$ between $\pm 14 \mathrm{~T}$ at $0.3 \mathrm{~T} / \mathrm{min}$ while stabilizing the temperature $T$ to within $\pm 5 \mathrm{mK}$.

Freshly cleaved thin samples were used for studying the surface transport in BSTS. As shown in Fig. 1(c), $\rho_{x x}$ in BSTS sharply increases upon lowering temperature from $300 \mathrm{~K}$, which is characteristic of an insulator, but it saturates below $\sim 100 \mathrm{~K}$ due to the metallic sur- 

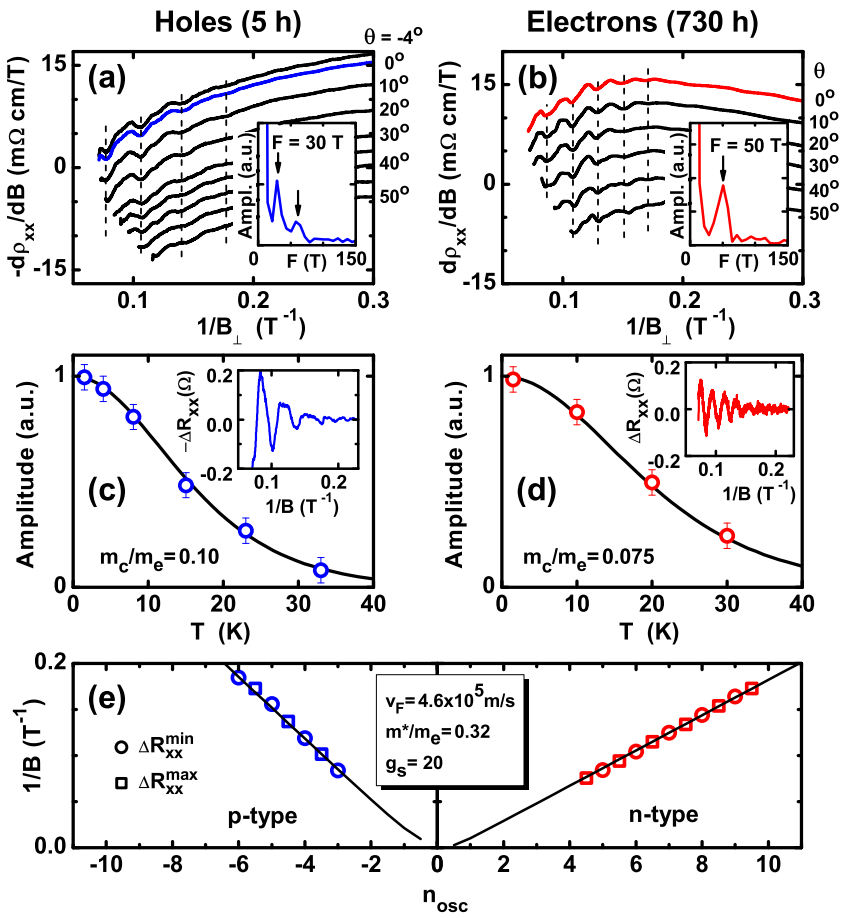

FIG. 2: (Color online) (a,b) $d \rho_{x x} / d B$ vs $1 / B_{\perp}$ measured in tilted magnetic fields in the $p$ state $(5 \mathrm{~h})$ and the $n$ state $(730$ h). All curves are shifted for clarity. Insets show the Fourier transforms of the data at $\theta=0^{\circ}$. (c,d) Temperature dependences of $\mathrm{SdH}$ amplitudes for $\theta=0^{\circ}$ shown in (a) and (b) and their theoretical fittings. Insets show $\Delta R_{x x}$ vs. $1 / B$ after subtracting a smooth background. (e) Landau-level fan diagram obtained from the oscillations in $\Delta R_{x x}$ measured at $T$ $=1.5 \mathrm{~K}$ and $\theta=0^{\circ}$; minima and maxima in $\Delta R_{x x}$ correspond to $n_{\mathrm{osc}}$ and $n_{\mathrm{osc}}+\frac{1}{2}$, respectively.

face transport as well as the bulk impurity-band (IB) transport [5]. This behavior is essentially the same as in BTS. What is peculiar in BSTS is that $\rho_{x x}(T)$ increases slowly with time and, furthermore, the low-temperature Hall coefficient $R_{H}$ changes sign with time in thin samples. As an example, Figs. 1(c) and (d) show $\rho_{x x}(T)$ and $R_{H}(T)$ data of a 30- $\mu$ m-thick BSTS sample, measured repeatedly in about one month. In contrast to the relatively small change in $\rho_{x x}$ [Fig. $\left.1(\mathrm{c})\right], R_{H}$ at lowtemperature exhibits rather drastic evolution [Fig. 1(d)] from positive to negative, whereas $R_{H}$ at high temperature is essentially unchanged with time. This suggests that the source of the time dependence resides in the surface channel. In passing, $R_{H}(T)$ above $150 \mathrm{~K}$ is positive and demonstrates an activated behavior [shown by the dashed line in Fig. 1(d)], signifying the thermal excitation of holes into the valence band with an effective activation energy of about $60 \mathrm{meV}$. This is similar to what we observed in BTS [5].

To understand the nature of the time evolution, the Shubnikov-de Haas ( $\mathrm{SdH})$ oscillations were measured in the aforementioned $30-\mu \mathrm{m}$-thick sample twice, $5 \mathrm{~h}$ after cleavage (called $p$ state) and $725 \mathrm{~h}$ later (called $n$ state), between which $R_{H}$ changed sign (the sample was kept at $300 \mathrm{~K}$ in air). In BSTS, the oscillations do not fade out even after long exposure to ambient atmosphere, as opposed to other TI materials like Sb-doped $\mathrm{Bi}_{2} \mathrm{Se}_{3}[10]$. The $\mathrm{SdH}$ oscillations were clearly observed in $\rho_{x x}(B)$ in our samples, but they were not clear in $\rho_{y x}(B)$, so the following $\mathrm{SdH}$ analysis is restricted to $\rho_{x x}(B)$. Figures 2 (a) and (b) show $d \rho_{x x} / d B$ for both the $p$ and $n$ states, plotted as a function of $1 / B_{\perp}(\equiv 1 / B \cos \theta)$, where $\theta$ is the angle between $B$ and the $C_{3}$ axis. Several equidistant maxima and minima are easily recognized, and importantly, the positions of maxima and minima depend solely on $B_{\perp}$, which signifies a $2 \mathrm{D}$ character of the observed oscillations. Insets show the Fourier transform of the oscillations taken at $\theta=0^{\circ}$. Two frequencies are clearly seen in the $p$ state, but the second one $(60 \mathrm{~T})$ is probably a harmonic of the primary frequency $F=30 \mathrm{~T}$. On the other hand, only the primary $F=50 \mathrm{~T}$ is seen in the $n$ state. The averaged Fermi wave number $k_{F}$ is obtained by using the Onsager relation $F=(\hbar c / 2 \pi e) \pi k_{F}^{2}$, resulting in $k_{F}=3.0 \times 10^{6} \mathrm{~cm}^{-1}$ and $3.9 \times 10^{6} \mathrm{~cm}^{-1}$ for the $p$ and $n$ states, respectively. This corresponds to the surface hole (electron) concentration of $7.2 \times 10^{11} \mathrm{~cm}^{-2}$ $\left(1.2 \times 10^{12} \mathrm{~cm}^{-2}\right)$ for a spin-filtered surface state. Fitting the standard Lifshitz-Kosevich theory [11] to the temperature dependences of the SdH amplitudes [Figs. 2(c) and $(\mathrm{d})]$ gives the cyclotron mass $m_{c}$ of $(0.10 \pm 0.01) m_{e}$ for holes and $(0.075 \pm 0.003) m_{e}$ for electrons $\left(m_{e}\right.$ is the free electron mass). Also, from the $B$-dependence of the $\mathrm{SdH}$ amplitudes [insets of Figs. 2(c) and (d)] one can obtain the scattering time $\tau$ of $5.8 \times 10^{-14} \mathrm{~s}\left(4.2 \times 10^{-14}\right.$ s) for holes (electrons) through the Dingle analysis.

From the measured values of $k_{F}$ and $m_{c}$, one obtains the effective Fermi velocity $v_{F}^{*}\left(\equiv \hbar k_{F} / m_{c}\right)$ of $3.5 \times 10^{5}$ $\mathrm{m} / \mathrm{s}$ and $6.0 \times 10^{5} \mathrm{~m} / \mathrm{s}$ for holes and electrons, respectively. Now we discuss that this difference between the two $v_{F}^{*}$ bears crucial information regarding the Dirac cone: If the surface state consists of ideal Dirac fermions, the Fermi velocity is independent of $\mathbf{k}$ and is particlehole symmetric. However, the energy dispersions of the surface states in Bi-based TI materials generally deviate from the ideal Dirac cone, and it can be described as 12 .

$$
E(k)=E_{D P}+v_{F} \hbar k+\frac{\hbar^{2}}{2 m^{*}} k^{2},
$$

where $E_{D P}$ is the energy at the Dirac point (DP), $v_{F}$ is the Fermi velocity at the DP, and $m^{*}$ is an effective mass that accounts for the curvature in $E(k)$. The effective Fermi velocity $v_{F}^{*}$ reflects the local curvature in $E(k)$ and can be expressed as $v_{F}^{*}(k)=(\partial E / \partial k) / \hbar=v_{F}+\hbar k / m^{*}$. The $p$ and $n$ states correspond to the situations where the Fermi energy $E_{F}$ is below or above the DP, respectively, and the time evolution of $R_{H}$ is a manifestation of the time-dependent change of the surface chemical potential. By using the $k_{F}$ and $v_{F}^{*}$ values obtained for the $p$ and 
$n$ states, we can solve simultaneous equations to obtain $v_{F}=4.6 \times 10^{5} \mathrm{~m} / \mathrm{s}$ and $m^{*}=0.32 m_{e}$. This mean that the time evolution of the transport properties allowed us to do a "spectroscopy" of the surface state to determine its dispersion, from which we can further estimate the position of $E_{F}$ to lie $80 \mathrm{meV}$ below (140 meV above) the DP in the $p(n)$ state. Finally, the mean free path $\ell_{s}$ $=v_{F}^{*} \tau$ is about $20 \mathrm{~nm}(40 \mathrm{~nm})$ and the surface mobility $\mu_{s}^{\mathrm{SdH}}=\left(e \ell_{s}\right) /\left(\hbar k_{F}\right)$ is about $1.0 \times 10^{3} \mathrm{~cm}^{2} / \mathrm{Vs}\left(9.8 \times 10^{2}\right.$ $\left.\mathrm{cm}^{2} / \mathrm{Vs}\right)$ in the $p(n)$ state.

To infer the Dirac nature of the surface state from the SdH oscillations [13, 14], Fig. 2(e) shows the Landaulevel (LL) fan diagram, which presents the values of $1 / B$ at the $n_{\text {osc }}$-th minima in the $\rho_{x x}$ oscillations as a function of $n_{\text {osc }}$ [14]. In the case of ideal Dirac particles, the diagram for holes (electrons) intersects the $n_{\mathrm{osc}}$-axis at -0.5 (0.5), reflecting the $\pi$ Berry phase $13-15]$. However, in recent $\mathrm{SdH}$ studies of TIs [5, 6, 10, 16 18] exact $\pi$ Berry phase has rarely been reported and this deviation has been a puzzle. The Zeeman coupling of the spins to the magnetic field can be a source of this discrepancy [10], and in addition, the deviation of $E(k)$ from the ideal linear dispersion also causes the Berry phase to shift from $\pi$ [14]. We therefore followed the scheme of Ref. [14] to see if the LL fan diagram obtained for BSTS can be understood by considering these additional factors: The solid lines in Fig. 2(e) show the theoretical LLs for the non-ideal Dirac fermions [14] with the band parameters already obtained $\left(v_{F}=4.6 \times 10^{5} \mathrm{~m} / \mathrm{s}\right.$ and $\left.m^{*}=0.32 m_{e}\right)$ and a surface $g$-factor $g_{s}=20$ (which is the sole fitting parameter). Those lines agree reasonably well with the experimental data for both the $p$ and $n$ states, supporting not only the Dirac nature of the observed surface state but also the conjecture that the holes and electrons reside on the same Dirac cone.

Now we discuss the mechanism for the time evolution of the transport properties in BSTS. At low temperature, the Fermi level is pinned to the IB in the bulk of the material [5], so the observed development of transport properties most likely comes from a change in the surface as schematically shown in Figs. 3(a) and (b). To understand the $p$ state where holes dominate the Hall response, one must assume that an upward band-bending occurs just after cleavage, putting the Fermi level below the DP and simultaneously creating a hole accumulation layer (AL) near the surface [Fig. 3(a)]. The air exposure apparently causes $n$-type doping on the surface as was reported for $\mathrm{Bi}_{2} \mathrm{Se}_{3}$ [19], leading to a downward band bending [Fig. 3(b)].

In the above picture, there must be three transport channels in the $p$ state: surface Dirac holes, bulk IB, and the surface AL due to the band bending. Hence, one may wonder if the $\mathrm{SdH}$ oscillations observed in the $p$ state might actually be due to the AL, rather than the Dirac holes. Fortunately, one can see that this is not the case, by analyzing the non-linear $B$ dependence of $\rho_{y x}$.
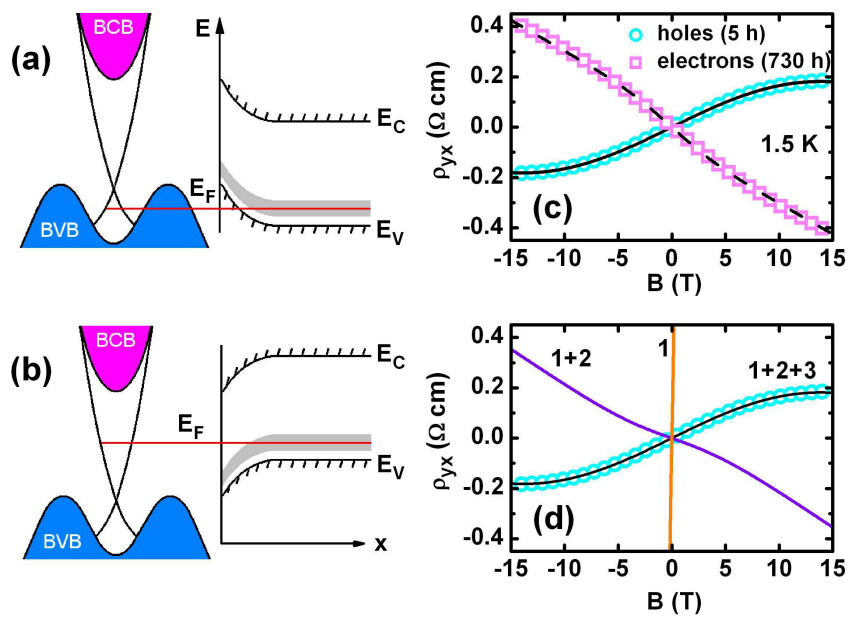

FIG. 3: (Color online) (a,b) Schematic picture of the bulk and surface states and the surface band bending for the $p$ and $n$ states, respectively. $E_{C}\left(E_{V}\right)$ is the energy of the conductionband bottom (valence-band top), and the shaded band depicts the impurity band. (c) $\rho_{y x}(B)$ data measured at $1.5 \mathrm{~K}$ in the $p$ and $n$ states. The dashed (solid) line is the fitting of $\rho_{y x}(B)$ in the $n$-state ( $p$-state) using the two-band (three-band) model. (d) Decomposition of the three-band $\rho_{y x}(B)$ fitting in the $p$ state (see text).

In the following, we discuss the analyses of the $\rho_{y x}(B)$ data, starting from the simpler case of the $n$ state.

As in BTS [5], the $\rho_{y x}(B)$ curves in the $n$ state of BSTS can be well fitted with a simple two-band model described in Ref. [5]. The dashed line in Fig. 3(c) is a result of such fitting to the $1.5-\mathrm{K}$ data, where the fitting parameters are the bulk electron density $n_{b}=2.3 \times 10^{16} \mathrm{~cm}^{-3}$, the bulk mobility $\mu_{b}=190 \mathrm{~cm}^{2} / \mathrm{Vs}$, and the surface mobility $\mu_{s}$ $=1250 \mathrm{~cm}^{2} / \mathrm{Vs}$ (the surface electron density was fixed at $1.2 \times 10^{12} \mathrm{~cm}^{-2}$ from the $\mathrm{SdH}$ data). These parameters give the residual bulk conductivity $\sigma_{b}$ of $0.73 \Omega^{-1} \mathrm{~cm}^{-1}$, and the surface contribution to the total conductance can be estimated as $G_{s} /\left(G_{s}+\sigma_{b} t\right) \approx 0.1$, where $G_{s} \approx$ $2.4 \times 10^{-4} \Omega^{-1}$ is the sheet conductance of the surface and $t=30 \mu \mathrm{m}$ is the thickness.

In the $p$ state, the AL must also be taken into account, so we tried a three-band analysis in which we assumed that the bulk carriers are the same as in the $n$ state. The solid line in Fig. 3(c) shows a result of the fitting to the $1.5-\mathrm{K}$ data, where the fitting parameters are the AL mobility $\mu_{s^{\prime}}=770 \mathrm{~cm}^{2} / \mathrm{Vs}$, the AL sheet conductance $G_{s^{\prime}}=2.2 \times 10^{-3} \Omega^{-1}$, and the Dirac-hole mobility $\mu_{s}=1170 \mathrm{~cm}^{2} / \mathrm{Vs}$ (the Dirac hole density was fixed at $7.2 \times 10^{11} \mathrm{~cm}^{-2}$ from the $\mathrm{SdH}$ data). To understand the relative importance of the three channels, it is instructive to examine the individual contributions to the total $\rho_{y x}$ : As shown in Fig. $3(\mathrm{~d})$, the putative $\rho_{y x}(B)$ due solely to the surface Dirac holes (curve 1) is strongly modified when the residual bulk contribution is added (curve $1+2$ ), but it is still very different from the mea- 


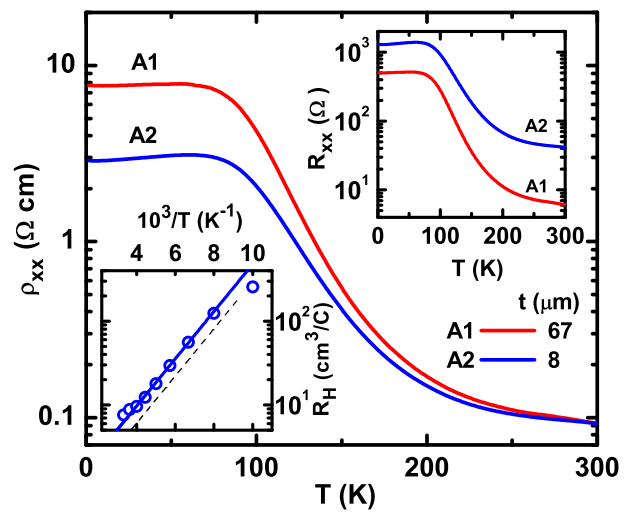

FIG. 4: (Color online) Temperature dependences of $\rho_{x x}$ in $0 \mathrm{~T}$ measured in a BSTS sample before and after reducing its thickness. Upper inset shows the plot of $R_{x x}(T)$ for the same set of data. Lower inset shows the high-temperature activation behavior in $R_{H}(T)$ for the $8-\mu \mathrm{m}$ sample, which is compared with the similar behavior observed in the $30-\mu \mathrm{m}$ sample [Fig. 1(d) inset] shown as a dashed line.

sured $\rho_{y x}(B)$; only when the third contribution of the AL is added (curve $1+2+3$ ), the $\rho_{y x}(B)$ behavior is satisfactorily reproduced.

Based on the above analysis, one can see that it is impossible to interpret the $\mathrm{SdH}$ oscillations in the $p$ state to originate from the $\mathrm{AL}$ : If the $\mathrm{SdH}$ oscillations were due to the $\mathrm{AL}$, the third transport channel must be the surface Dirac holes; however, the sheet carrier density $n_{s^{\prime}}$ of the third channel is estimated as $n_{s^{\prime}}=G_{s^{\prime}} /\left(e \mu_{s^{\prime}}\right) \approx$ $1.8 \times 10^{13} \mathrm{~cm}^{-2}$, which is too large for Dirac holes for which $k_{F} \lesssim 5 \times 10^{6} \mathrm{~cm}^{-1}$ [20] and hence $n_{s^{\prime}}$ must be $\lesssim$ $2 \times 10^{12} \mathrm{~cm}^{-2}$. Therefore, one can safely conclude that the SdH oscillations are due to the Dirac holes.

Lastly, we show that the surface conductance in BSTS can be reasonably estimated by simply changing the thickness. Figure 4 shows the temperature dependences of $\rho_{x x}$ of a different sample, measured first in $67-\mu \mathrm{m}$ thickness, and later cleaved down to $8-\mu \mathrm{m}$ thick 21]. The overall behavior of $\rho_{x x}(T)$ is similar between the two curves, but a striking difference lies in their lowtemperature saturation values $\rho_{1}^{\text {sat }}$ (for $67 \mu \mathrm{m}$ ) and $\rho_{2}^{\text {sat }}$ (for $8 \mu \mathrm{m}$ ): it is lower in the thinner sample, implying a larger relative surface contribution. Note that the resistance $R_{x x}$ duly increases upon reducing the thickness, as shown in the upper inset of Fig. 4. Instructively, the difference in $\rho_{x x}$ disappears at high temperature where the bulk conduction dominates. From the observed difference in $\rho_{i}^{\text {sat }}$ one can estimate the surface and bulk contributions to the total conductivity by using $\rho_{i}^{\text {sat }}=\left[\left(G_{s} / t_{i}\right)+\sigma_{b}\right]^{-1}$. We obtain $G_{s} \approx 1.8 \times 10^{-4} \Omega^{-1}$ and $\sigma_{b} \approx 0.1 \Omega^{-1} \mathrm{~cm}^{-1}$, and this $\sigma_{b}$ is much smaller than that in the $30-\mu \mathrm{m}$-thick sample. This is probably because the number of acceptors is smaller in this second sample [22], as can be inferred in the high-temperature $R_{H}$ be- havior [lower inset of Fig. 4]. The obtained values of $G_{s}$ and $\sigma_{b}$ allows us to calculate the fraction of the surface contribution to the total conductance, $G_{s} /\left(G_{s}+\sigma_{b} t_{i}\right)$, for the $67-$ and $8-\mu \mathrm{m}$ thick samples to be 0.2 and 0.7 , respectively. Therefore, when the thickness of a BSTS sample is reduced to $\lesssim 10 \mu \mathrm{m}$, one can achieve a bulk TI crystal where the topological surface transport is dominant over the bulk transport.

In summary, we show that one can achieve a surface-dominated transport in the new TI compound $\mathrm{Bi}_{1.5} \mathrm{Sb}_{0.5} \mathrm{Te}_{1.7} \mathrm{Se}_{1.3}$. The surface band bending and its time dependence makes it possible to observe the SdH oscillations of both Dirac holes and electrons, with which we could determine the surface band dispersion across the Dirac point. In addition, by analyzing the non-linear $\rho_{y x}(B)$ curves, we could identify the role of the surface accumulation layer in the transport properties. Obviously, this material offers a well-characterized playground for studying the topological surface state.

This work was supported by JSPS (NEXT Program and KAKENHI 19674002), MEXT (Innovative Area "Topological Quantum Phenomena" KAKENHI), and AFOSR (AOARD 10-4103).

[1] L. Fu and C. L. Kane, Phys. Rev. B 76, 045302 (2007).

[2] J. Moore, L. Balents, Phys. Rev. B 75, 121306(R) (2007).

[3] M.Z. Hasan, C. Kane, Rev. Mod. Phys. 82, 3045 (2010).

[4] X.L. Qi and S.C. Zhang, arXiv:1008.2026 1.

[5] Z. Ren et al. Phys. Rev. B 82, 241306(R) (2010).

[6] J. Xiong et al., arXiv:1101.1315

[7] Since the anti-site defects occur between $\mathrm{Bi}$ and $\mathrm{Te}$, reducing the $\mathrm{Te} / \mathrm{Se}$ ratio would suppress the anti-site; at the same time, Se in the outermost layer is prone to vacancy creations, which is suppressed by Sb doping.

[8] S. Nakajima, J. Phys. Chem. Solids 24, 479 (1963).

[9] The sample is suspended on gold wires in order to insure that both surfaces are in the same condition.

[10] J.G. Analytis et al., Nat. Phys. 10, 960-964 (2010).

[11] D. Shoenberg, Magnetic Oscillations in Metals (Cambridge University Press, Cambridge, 1984).

[12] D. Culcer et al., Phys. Rev. B 82, 155457 (2010).

[13] G. Mikitik, Yu. Sharlai, Phys. Rev. Lett. 82, 2147 (1999).

[14] A. A. Taskin and Y. Ando, arXiv:1103.3096.

[15] Y. Zhang et al., Nature (London) 438, 201 (2005).

[16] D.X. Qu et al., Science 329, 821 (2010).

[17] B. Sacépé et al., arXiv:1101.2352.

[18] C. Brüne et al., Phys. Rev. Lett. 106, 126803 (2011).

[19] J.G. Analytis et al., Phys. Rev. B 81, 205407 (2010).

[20] S.-Y. Xu et al., arXiv:1007.5111, D. Hsieh et al., Nature (London) 460, 1101 (2009).

[21] The cleaved 8 - $\mu$ m-thick sample showed a gradual increase in $\rho_{x x}$ similar to the $30-\mu$ m-thick sample, so we only used relaxed samples (in which the AL is absent) for the thickness-dependence study.

[22] Unfortunately the SdH oscillations were not observed in this sample, probably due to a lower surface mobility. 